\title{
A ONE-ONE SELECTION THEOREM
}

\author{
H. SARBADHIKARI
}

\begin{abstract}
Let $X, Y$ be Polish spaces without isolated points and $B \subseteq X \times Y$ a Borel set such that $\left\{x: B_{Y}\right.$ is nonmeager $\}$ is comeager in $X$ and $\left\{y: B^{y}\right.$ is nonmeager $\}$ is comeager in $Y$. There is a comeager Borel $E \subseteq X$, a comeager Borel $F \subseteq Y$ and a Borel isomorphism $f$ from $E$ onto $F$ such that graph of $f \subseteq B$.
\end{abstract}

1. Introduction. In [3] Mauldin proved that if $B \subset[0,1] \times[0,1]$ is a Borel set such that $\lambda\left\{x: \lambda\left(B_{x}\right)>0\right\}=1$ and $\lambda\left\{y: \lambda\left(B^{y}\right)>0\right\}=1$, where $\lambda$ is the Lebesgue measure, then there exist Borel sets $E$ and $F$ of full measure and a Borel isomorphism $f$ from $E$ onto $F$ such that the graph of $f \subseteq B$. Our main theorem is a category analogue of this. Throughout this paper, $X, Y$ are taken to be Polish spaces without isolated points.

2. The main result. Our main theorem reads

Let $B \subset X \times Y$ be a Borel set such that $\left\{x: B_{x}\right.$ is nonmeager $\}$ is comeager in $X$ and $\left\{y: B^{y}\right.$ is nonmeager $\}$ is comeager in $Y$. Then there is a comeager Borel $E \subseteq X$, a comeager Borel $F \subseteq Y$ and a Borel isomorphism $f$ from $E$ onto $F$ such that the graph of $f=\{(x, y): y=f(x)\} \subseteq B$.

Our proof is analogous to that in [3] where several subsidiary results are proved, leading to the main theorem.

Theorem 1. Let $X=Y=[0,1], B \subseteq X \times Y$ be a Borel set such that $\left\{x: B_{x}\right.$ is comeager $\}$ is comeager in $X$. There is a comeager Borel $E \subseteq X$, a meager Borel $F \subseteq Y$ and a Borel isomorphism from $E$ onto $F$ with graph $f \subseteq B$.

Proof. Fix an open base $\left\{U_{n}: n=1,2, \ldots\right\}$ for $Y$ consisting of nonempty intervals.

Note that $B$ is comeager in $X \times Y$. Hence there exist dense open sets $V_{1} \supseteq V_{2} \supseteq$ $\cdots$ in $X \times Y$ with $\cap_{n} V_{n} \subseteq B$.

By induction on $n$, we define a sequence of Borel sets $\left\{H_{n}: n=1,2, \ldots\right\}$ such that for all $n$

(1) $H_{n+1} \subseteq H_{n} \subseteq V_{n}, \ldots$

(2) There exist a sequence $\left\{B_{n i}: i=1,2, \ldots\right\}$ of pairwise disjoint nonmeager $G_{\delta}$ sets in $X$ with $B_{n i} \subseteq\left(k_{i} / 2^{n},\left(k_{i}+1\right) / 2^{n}\right)$ for some integer $k_{i}$ and $\bigcup_{i} B_{n i}$ comeager

Received by the editors November 6, 1984 and, in revised form, May 20, 1985

1980 Mathematics Subject Classification. Primary 04A15; Secondary 03E15, 54C65.

Key words and phrases. Comeager set, Borel isomorphism, graph of a function. 
in $X$; a sequence $\left\{\left[a_{n i}, b_{n i}\right]: i=1,2, \ldots\right\}$ of pairwise disjoint closed intervals of length $>0$ and $\leqslant 1 / 2^{n}$ and a nonempty open $W_{n} \subseteq U_{n}$ with $W_{n} \cap \cup_{i}\left[a_{n i}, b_{n i}\right]=\varnothing$ such that $H_{n}=\bigcup_{i} B_{n i} X\left(a_{n i}, b_{n i}\right)$.

(3) For all $x, \bar{H}_{n+1 x} \subseteq H_{n x}$.

Now $\bigcap_{n} H_{n}$ is the graph of the required function $f$.

Construction of $H_{n}$. Suppose $H_{m}$ has been defined and equals $\cup_{i} B_{m i} X\left(a_{m i}, b_{m i}\right)$ as in condition (2). For each $i$, we define $H_{m+1}^{i}$ so that $\bigcup_{i} H_{m+1}^{i}=H_{m+1}$.

Fix $i$. Note that $V_{m+1} \cap\left(B_{m i} \times\left(a_{m i}, b_{m i}\right)\right)$ is comeager in $B_{m i} X\left(a_{m i}, b_{m i}\right)$. Hence by the Kuratowski-Ulam theorem, $\left\{y \in\left(a_{m i}, b_{m i}\right): V_{m+1}^{y} \cap B_{m i}\right.$ is comeager in $\left.B_{m i}\right\}$ is comeager in $\left(a_{m i}, b_{m i}\right)$. Pick $y_{1}, y_{2}$ from this set with $y_{1}<y_{2}$. Let $M>$ $\operatorname{maximum}\left\{1 /\left(y_{2}-y_{1}\right), 1 / l, 2^{m}\right\}$, where $l=\max \left(\right.$ length $\left.U_{m+1} \cap\left(a_{m i}, b_{m i}\right), 1\right)$ and

$$
\begin{aligned}
A_{n}^{i}=\left\{x \in B_{m i}:\left[y_{1}-\frac{1}{4 n}, y_{1}+\frac{1}{4 n}\right] \cup\left[y_{2}-\right.\right. & \left.\frac{1}{4 n}, y_{2}+\frac{1}{4 n}\right] \\
& \left.\subseteq V_{m+1 x} \cap\left(a_{m i}, b_{m i}\right)\right\}, \quad n \geqslant M .
\end{aligned}
$$

Then $A_{n}^{i}$ is coanalytic and $\bigcup_{n \geqslant M} A_{n}^{i}=V_{m+1}^{v_{1}} \cap V_{m+1}^{v_{2}} \cap B_{m i}$ is a comeager Borel set in $B_{m i}$.

Find pairwise disjoint Borel sets $B_{n}^{i} \subset A_{n}^{i}, n \geqslant M$, with $\cup_{n} B_{n}^{i}=\bigcup_{n} A_{n}^{i}$. Put $C_{n}^{i}=B_{n}^{i} \cap\left(k_{i} / 2^{n},\left(2 k_{i}+1\right) / 2^{n+1}\right), D_{n}^{i}=B_{n}^{i} \cap\left(\left(2 k_{i}+1\right) / 2^{n+1},\left(k_{i}+1\right) / 2^{n}\right)$. Note that by possibly ignoring a meager set, we can suppose $C_{n}^{i}$ and $D_{n}^{i}$ to be nonmeager $G_{\delta}$ sets in $X$. Put

$$
H_{m+1}^{i}=\bigcup_{n \geqslant M}\left(C_{n}^{i} X\left(y_{1}-\frac{1}{4 n}, y_{1}-\frac{1}{4 n+1}\right) \cup D_{n}^{i} X\left(y_{2}-\frac{1}{4 n}, y_{2}-\frac{1}{4 n+1}\right)\right) \text {. }
$$

To construct $H_{1}$, use $V_{1} \cap X \times(0,1)$ as a comeager open set in $X \times Y$ and proceed as above.

Corollary. The previous theorem is true even when $X$ and $Y$ are arbitrary Polish spaces without isolated points.

Proof. Since the irrationals are homeomorphic to a comeager $G_{\delta}$ subset of $[0,1]$, the result is true if $X=Y=$ irrationals.

Now any Polish space without isolated points contains a comeager $G_{\delta}$ set homeomorphic to irrationals. Thus the result is true for $X, Y$ such spaces.

THEOREM 2. Let $B \subseteq X \times Y$ be such that $\left\{x: B_{x}\right.$ is nonmeager $\}$ is comeager. Then there is a comeager Borel $E \subseteq X$, a meager Borel $F \subseteq Y$ and a Borel isomorphism $f$ on $E$ onto $F$ such that graph $f \subseteq B$.

Proof. Let $U_{1}, U_{2}, \ldots$ be a countable open base for $Y$. Let $B_{n}^{*}=\left\{x: B_{x} \cap U_{n}\right.$ is comeager in $\left.U_{n}\right\}$ and $A_{n}=B_{n}^{*}-\bigcup_{m<n} B_{m}^{*}$. $A_{n}$ is Borel for all $n$ and $\bigcup_{n} A_{n}$ is comeager in $X$.

By ignoring a meager set if necessary, we can suppose that each $A_{n}$ is a nonmeager $G_{\delta}$. By induction on $n$, we define $f_{n}$ on $E_{n} \subseteq A_{n}$. We then define $f(x)=f_{n}(x)$ for $x \in E_{n}$. 
Suppose $f_{k}, k \leqslant m$, has been defined and range $f_{k} \subseteq$ a meager $F_{\sigma}$ set, say $F_{k} \subseteq U_{k}$. Put

$$
B_{m+1}=A_{m+1} X\left(U_{m+1}-\bigcup_{i=1}^{m} F_{i}\right) \cap B .
$$

$B_{m+1}$ is a Borel subset of $A_{m+1} X\left(U_{m+1}-\bigcup_{i=1}^{m} F_{i}\right)$ and $\left\{x: B_{m+1 x}\right.$ is comeager (in $\left.\left.U_{m+1}-\bigcup_{i=1}^{m} F_{i}\right)\right\}=A_{m+1}$. By applying the previous result, get a comeager $G_{\delta} E_{m+1}$ in $A_{m+1}$ and a Borel isomorphism $f_{m+1}$ on $E_{m+1}$ into $U_{m+1}-\bigcup_{i=1}^{m} F_{i}$ such that range $f_{m+1}$ is meager.

If $f(x)=f_{n}(x)$ for $x \in E_{n}, f$ is a Borel isomorphism on $\bigcup_{n} E_{n}$ into $\bigcup_{n} F_{n}$. Thus domain $f$ is comeager and the range is meager.

Proof of the MAIN TheOrem. Find Borel sets $E_{1} \subseteq X, F_{1} \subseteq Y$ such that $E_{1}$ is comeager, $F_{1}$ is meager and there is a Borel isomorphism $h$ from $E_{1}$ onto $F_{1}$ satisfying graph $h \subseteq B$.

Find Borel sets $G \subseteq Y, H \subseteq X$ such that $G$ is comeager, $H$ is meager and there is a Borel isomorphism $g$ from $G$ onto $H$ satisfying $\{(x, y): x=g(y)\} \subseteq B-X \times F_{1}$.

Define $f$ on $E_{1} \cup H$ by

$$
\begin{aligned}
f(x) & =g^{-1}(x) & & \text { if } x \in H, \\
& =h(x) & & \text { if } x \in E_{1}-H .
\end{aligned}
$$

Putting $E=E_{1} \cup H, F=$ range $f$, we get the result.

REMARKS. In [3] Mauldin raises some interesting questions of which the following are still open to our knowledge.

1. If $B \subseteq[0,1] \times[0,1]$ is a Borel set with $B_{x}, B^{y}$ of positive Lebesgue measure for all $x$ and $y$, is there a Borel isomorphism of $[0,1]$ onto $[0,1]$ whose graph is a subset of $B$ ?

2. Is the category analog of the above true?

ACKNOWLEDGMENT. I am grateful to Dr. R. D. Mauldin for bringing this problem to my attention and to Dr. S. M. Srivastava for simplifying the proof.

\section{REFERENCES}

1. S. Graf and R. D. Mauldin, Measurable one-to-one selections and transition kernels, Amer. J. Math. (to appear).

2. K. Kuratowski, Topologv, Vol. I, Academic Press, New York; PWN, Warsaw, 1966.

3. R. D. Mauldin, One-to-one selections-marriage theorems, Amer. J. Math. 104 (1982), 823-828.

Indian Statistical Institute, Division of Theoretical Statistics and Mathematics, 203 BarRACKPORE TRUNK ROAD, CALCUTTA 700 035, INDIA 